\title{
Inbred Strain
}

National Cancer Institute

\section{Source}

National Cancer Institute. Inbred Strain. NCI Thesaurus. Code C28422.

A strain that has been maintained by sibling (sister $\mathrm{x}$ brother) matings for 20 or more consecutive generations. (from JAX Strain Information) 